\title{
Embotelladoras ARCA Uses Operations Research to Improve Territory Design Plans
}

\author{
J. Fabián López-Pérez \\ Embotelladoras ARCA \\ Monterrey, Mexico \\ e-mail: fabian.lopez@arcacontal.com
}

Roger Z. Ríos-Mercado ${ }^{1}$

Graduate Program in Systems Engineering

Universidad Autónoma de Nuevo León, Mexico

e-mail: roger.rios@uanl.edu.mx

June 2011

${ }^{1}$ Corresponding author 


\begin{abstract}
Embotelladoras ARCA was formed in 2001 by integrating three of the oldest bottlers in Mexico and became the second largest bottler of Coca-Cola products in Latin America. The company distributes its products in the northern region of Mexico and, since 2008, in the north of Argentina and Ecuador. The company have soft-drink sales of more than 1.2 billion unit cases and ranks as the third-largest Coca-Cola bottler in the world. The large size of the market and the relevance of a number of problems faced by the company motivate the use and application of operations research models and techniques One of the most relevant problems the company faces is that of how to segment or partition their customers into clusters or territories to accomodate for a better handling of marketing and distribution decisions. This territory design is not entirely arbitrary since it must satisfy several planning requirements such as territory compactness, territory connectivity, territory balancing, and similarity with existing design. Before 2009, these units were defined by "experience" without quantitative tools giving more weight to the territory compactness criterion. This led to a number of undesirable issues such as highly unbalanced territories, that is, the plans ended up with a large disparity in size with respect to both number of customers and total product sales. This imbalance had a negative effect among company workers since each territory (customer order capturing by sales associates, product routing by truck drivers, and so on) is handled by a different team. In this paper, we apply operations research methods to determine better configurations of the territorial units to ensure that each formed territory is relatively similar in size with respect to both number of customers and total product sales while ensuring some other important planning requirements and maximizing territory compactness. The usage of this methodology has resulted in many important benefits for the company, in particular, it has had a significant improvement with respect to the territory imbalances improving from 30 to $5 \%$. We highlight some other side benefits resulting from this approach. The company has adopted this proposed tool to make their territory design decisions.
\end{abstract}

Keywords: Bottled beverage distribution; Commercial districting; Mixed-integer programming model; Operations research; Territorial units. 


\section{Introduction}

Embotelladoras ARCA was formed in 2001 by integrating three of the oldest bottlers in Mexico and became the second largest bottler of Coca-Cola products in Latin America. The company distributes its products in the northern region of Mexico and, since 2008, in the north of Argentina and Ecuador. ARCA also produces and distributes branded salty snacks Bokados. Embotelladoras Arca merged with Grupo Continental in January 2011 in a \$ 2.3 USD billion exchange of stock and the resulting conglomerate is called Embotelladoras ARCA-Continental (or Arca-Contal:, http://www.arcacontal.com/.) ArcaContal is a company dedicated to production, distribution and sales of snacks and soft drinks brands. The company have soft-drink sales of more than 1.2 billion unit cases and ranks as the third-largest Coca-Cola bottler in the world. Arca-Contal sells Ciel bottled water, tea, energetic drinks, and snacks, as well as the usual Coke brands. The company has its headquarters in Monterrey Mexico. The large size of the market and the relevance of a number of problems faced by the company motivate the use and application of operations research models and techniques

One of the most relevant problem the company faces is that of how to segment or partition their customers into clusters or territories to accomodate for a better handling of marketing and distribution decisions. In esence, this is a commercial Territory Design Problem (TDP). Commercial TDP may be viewed as the problem of grouping basic units (i.e. city blocks, zip codes, or individual customers) into subsets according to specific planning criteria. These subsets are known as territories or districts. There are some other spatial constraints as part of the geographic definition of the problem. Depending on the context of the problem, the concept "territory design" may be used as equivalence to "districting". Districting is a truly multidisciplinary research which includes several fields such as geography, political science, public administration, and operations research. However, all these problems have in common the task of subdividing the region under planning into a number of territories, subject to some planning constraints. Indeed, territory design problems emerge from different type of real world applications. We can mention pick up and delivery applications, waste collection, school districting, sales workforce territory design and even some others related to geo-political concerns. Most public services including hospitals, schools, postal delivery, etc., are administered along territorial boundaries. We can mention either economic or demographic issues that may be taken in consideration for setup a balanced territory. On this work we are going to focus our study in the city of Monterrey, Mexico. In the distribution industry, a TDP is motivated by changes around the customers served by a given route.

As each territory is to be served by a single resource, it makes sense to use some planning 
criteria to balance the quantity of customers, product demand, and workload required by the dispatchers or truck drivers to cover each territory. Moreover, it is often required to balance the demand among the territories in order to delegate responsibility fairly. To this end, the firm wishes to partition the city area into disjoint territories that are suitable for their commercial purposes. In particular, given a set of city blocks or basic units (BUs), the firm wants to create a specific number of territories according to some planning criteria such as (i) compactness: customers as close to each other as possible, (ii) balancing with respect to each of two activity measures (number of customers and product demand), (iii) territory connectivity: such that a truck assigned to a territory can deliver the goods without leaving the territory, (iv) disjoint BU assignment: that avoids assigning a specific subset of customers to the same territory, and (v) similarity with existing plan for a subset of BUs. In other words, the main objective of TDP is to group the customers into manageable sized territories in order to guarantee that BUs assigned to a territory are relatively close to each other and meeting the aforementioned planning criteria.

To address this territory design problem, we derive a mixed-integer linear programming (MILP) model, and develop a solution framework based on the iterative solution of an associated MILP model with a cut generation strategy. This has been implemented with an off-the-shelf modeling and optimization suite. The model and solution method are integrated into an interactive and user-friendly Geographic Information System (GIS) application, named MAPINFO (c). This paper describes and illustrates the potential of the proposed approach as an easy to use decision tool in the context of a case study developed on a large soft drink company that operates in the city of Monterrey, Mexico. In the following sections, we (1) describe in detail the problem we are facing, (2) describe an overview of other approaches to commercial districting problems, (3) present a general description of the solution framework, (4) illustrate the usefulness of the approach with a case study, and (5) highlight the practical benefits that resulted from this work.

\section{Overview of Related Work}

Depending on the context of the problem, Territory Design (TD) may be used as equivalent to Districting which is a truly multidisciplinary research field which includes several areas such as geography, political science, public administration and Operations Research, as well. We can generalize that TD is common to all applications that operate within a group of resources that need to be assigned in an optimal way in order to subdivide the work area into balanced regions of responsibility. We can mention pick up and delivery applications, waste collection, political districting, school districting, sales workforce territory design, and even some others related to geopolitical concerns. Most public services 
including hospitals, schools, and so on, are managed along territorial boundaries. We can mention either economic or demographic issues that may be considered for setting-up a well balanced territory.

For excellent reviews on models, algorithms, and applications on territory design and districting in the past few years, the reader is referred to the recent works by Kalcsics, Nickel, and Schröder [3], Duque, Ramos, and Suriñach [2], and Zoltners and Sinha [11]. These papers given an up to date account of the most important and relevant applications of districting problems in general.

In this section, we highlight the most relevant work on commercial territory design, that are most closely related to the problem of interest. The first work on commercial territory design was due to Vargas-Suarez, Ríos-Mercado, and López [8], who address a problem with a variable number of territories, aiming at optimizing the territory balancing with respect to three activity measures (number of customers, product demand, and workload). No compactness criterion was considered. A basic metaheuristic based on GRASP was developed and tested in a few instances obtaining relatively good results. Ríos-Mercado and Fernández [4] studied the problem by considering compactness and connectivity but with no joint or disjoint assignment constraints. They used the objective function of the $p$-Center Problem $(p \mathrm{CP})$ for modeling territory dispersion. In that work, the authors proposed and developed a reactive GRASP algorithm for handling large instances. They evaluated their algorithm on 500- and 1000-node instances with very good results. More recently, SalazarAguilar et al. [5] develop an exact optimization scheme for solving the TDP with double balancing and connectivity constraints. They used their framework for solving models with both types of dispersion functions: the one based on the $p \mathrm{CP}$ and the one based on the $p$-Median Problem $(p \mathrm{MP})$. They observed that models with a $p \mathrm{MP}$ objective function had a tighter LP relaxation and therefore solved faster than the ones using a $p \mathrm{CP}$ objective. Furthermore, they also observed that solutions obtained from the relaxation of the $p \mathrm{MP}$ based models had a very high degree of connectivity. Still, the largest instance they could solve for the $p \mathrm{MP}$ based models was about 150 BUs. In our approach, we use a similar framework than the one they used in their work, except that we will be focusing in the allocation phase aiming at significantly larger instances. More recently, several approaches have been developed for multiobjective versions of the commercial TDP, including both exact optimization approaches [6] and metaheuristic methods [7].

Our model has features that extend previous models and that have not been addressed before in the commercial territory design context such as the disjoint assignment constraints and similarity with existing plan. Besides, the mathematical structure of our problem is indeed different from earlier models that make previous approaches not applicable for our specific model. 


\section{Territory Design Application at Embotelladoras ARCA}

TDP models and solutions are case-specific, since each of them has its own constraints and objectives, making it practically impossible to create a general purpose algorithm that can be applied to all types of instances. When reviewing the literature, one can observe that only a few papers consider territory design problems independently from a concrete practical background. Hence the tendency in operations research to separate the model from the application and establish the model itself as a selfcontained topic of research is not observed. Therefore, we introduce a real business model applied to territory design and present a solution framework tailored for this particular application.

The territory design problem can be defined as the process of grouping small geographic areas, i.e. basic areas or units (BUs), into clusters or territories. The new geographic clustered areas are called territories. It is required that each basic area should be contained in exactly one territory. Moreover, we require compactness and connectivity for the territories constructed. Indeed, contiguity can be defined as a territory where it is possible to travel between every pair of basic units by following a path contained in the territory, in other words, the basic areas that conform a territory have to be geographically connected. It is easy to understand, that in order to obtain connected territories, explicit neighborhood information for the basic areas is required. Our problem definition includes two measurable attributes or activities for each basic area. Three activity measures are used for each basic area: (i) number of customers, and (ii) sales volume or product demand. The activity measure of a given territory is the total of all activity measure of the individual basic units belonging to it. As stated before, it is required that each territory is balanced with respect to each activity, that is that territories are similar in size. Balancing number of customer, for instance, implies a fair work distribution among the sales people that handles the indvidual orders. Balancing product demand implies a fair distribution among the truck drivers. It is interesting to point out, that only a few authors consider more than one criterion simultaneously for designing balanced territories (Deckro [1], Zoltners [9], Zoltners and Sinha [10]).

Finally, the number of territories $p$ to be constructed is known in advance. Our problem definition includes some prescribed and/or forbidden territories. That means that from the beginning we already have some basic areas which are required to be assigned to a specific territory (called joint assignment constraints). Furthermore, there are other basic areas which are not allowed to be assigned to the same territory (called disjoint assignment constraints). As can be verified, all these features could be easily extended to consider some territories that may already exist at the beginning of the planning process. That means that our method could be prepared to take the already existing territories into 
account and then add additional basic areas to them. This modeling feature could be applied to consider geographical obstacles, e.g. rivers and mountains. We can generalize that the territory design problem is common to all applications that operate with a group of resources that need to be assigned in order to subdivide the work area into a balanced regions of responsibility. The problem can be summarized as follows: partition the set $V$ of basic areas into $p$ territories which satisfy the specified planning criteria such as balance, compactness, connectivity, disjoint assignment, and similarity with existing BU assignment.

The problem specifications can be summarized as follows:

- Given a set of BUs (city blocks) for delivering bottled beverages, we need to partition this set into a given number of disjoint territories.

- Each BU must be fully assigned to a single territory. It is not allowed to split BUs. That is, for each BU, the route that delivers product type 1, for instance, should be the same as the one that is responsible for delivering product type 2 .

- For each BU, the following information is known with certainty: location coordinates (from the firm GIS), number of customers, product demand or sales volume measured by number of 12-bottle boxes.

- The firm wants to design territories that are balanced (similar in size) with respect to each of the the two different activity measures in every BU. That is, the total number of customers and product demand assigned to each territory should be fairly distributed among the territories.

- Territories must be connected, that is, for any two BUs belonging to the same territory there must be a path connecting them totally contained in the territory.

- There is some pre-defined pairs of BUs that are required to be assigned to the same territory as much as possible. This is called similarity with existing plan.

- In a similar fashion, there are some predefined pairs of BUs that must be assigned to different territories. We called these disjoint assigment constraints.

- The goal of the design is to obtain territories that are as compact as possible, that is the BUs in a given territory must be as close to each other as possible and whose assigment includes as much as possible the similarity with existing subset of BUs.

This problem is modeled as a mixed-integer program, which is included in the Appendix. 


\section{Overview of Solution Framework}

In this section we present a solution strategy for solving the Allocation Model (AM) given in the Appendix. One main difficulty in the exponential number of connectivity constraints (5), which implies it is practically impossible to write them out explictly. Therefore, we consider instead the relaxation AMR of AM that consists of relaxing these connectivity constraints. The basic idea of our method is to solve model AMR and then check if the solutions obtained satisfy the connectivity constraints. To determine the violated connectivity constraints, a relatively easy separation problem is solved, and these cuts are added to model AMR. This procedure iterates until no additional connectivity constraints are found and therefore an optimal solution to model AM is obtained. This is guaranteed because the separation problem for identifying violated cuts is solved exactly. A general overview of the method is depicted in Figure 1.

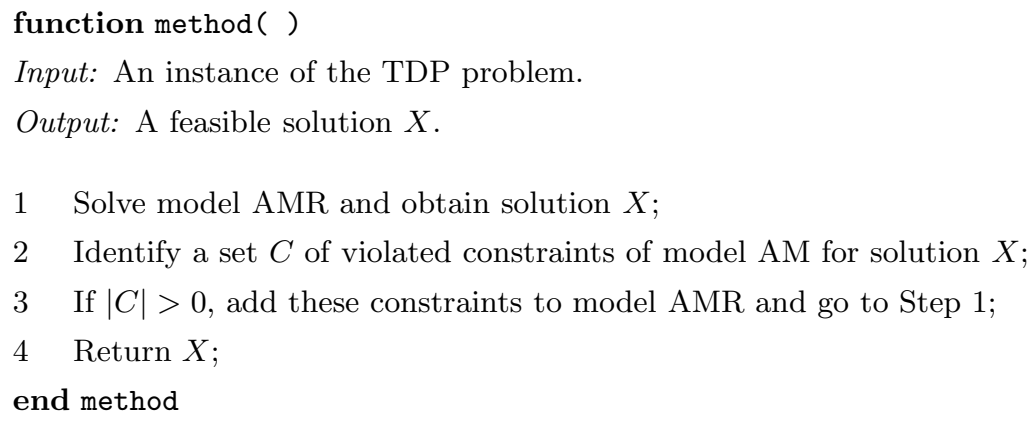

Figure 1: A pseudocode of solution procedure.

In Step 1, a branch-and-bound method is used (since we are not relaxing the integrality requirements of the binary variables). This approach is motivated by the fact that model AMR can be solved optimally by current branch-and-bound methods relatively fast for relatively large instances. For instance, 2000-node instances can be solved in a few seconds of CPU time in a PC. In addition, identifying and generating the violated cuts in Step 2 can also be done in polynomial time, so the overall procedure may be suitable as long as the number of iterations needed to reach optimality is not too large. The algorithm delivers an optimal solution to model AM.

The fact we are assuming a fixed set of centers is further exploited to develop several algorithmic strategies for speeding up convergence. Some of the strategies that have been implemented are: (i) Variable fixing at preprocessing that allows to identify and fix at 1 (0) BUs that are relative far away (closed to) territory centers; and (ii) Strenghtening of connectivity constraints by adding to the relaxed model (a polinomial number of) some connectivity constraints that prevent forming unconnected territories of size 1 (which are 
the most commonly found in a disconnected solution from the relaxed model).

\section{Case Study}

We implement our solution framework by using the X-PRESS MIP Solver and language capabilities from FICO ${ }^{\mathrm{TM}}$ (Fair Isaac, Dash Optimization before). The method was executed on a PC with 2 Intel Core processors at $1.4 \mathrm{GHz}$ and Win XP operating system. For assessing the proposed method, and illustrate its usefulness, we use some real-world instances of 5000, and 50 territories.

According to our solution procedure, some input data is required as input to the solution method. The following tasks are performed to this end.

- A Geo-database layer with the set of points representing the BUs to be clustered into territories. To develop this database it was necessary to locate all customers using a GPS device. This data collection was accomplished by sales people by hand held equipments. All the customers on the city of Monterrey (about 65,000) were visited and points to every one of them were marked using a GPS device that received latitude and longitude coordinates.

- Eventually, by a very simple GIS application, all these customers can be aggregated into a number of 5000 BUs. Each of these BUs corresponds to a physical block in the city of Monterrey.

- An info-database layer containing the three activity measures (attributes) for each BU. As we mention before we have: (1) number of customers, (2) sales volume and (3) workload.

- The number of territories the end-user requires to construct for this study was set to $p=50$.

Before the development of this tool, one of the key issues was that of significant amount territory imbalance with respect to both number of customers and total product demand. The designs were constructed by hand based on "experience". The compactness criterion played a major role in this operation, but this yielded territories whose deviation from the ideal target were off by up to $30 \%$, which of course produced unrest among worker teams assigned to different territories. Now that this balancing requirement is explicitly modeled, one can achieve feasible designs whose deviation from the ideal target is within $10 \%$, and in some cases within $5 \%$. This of course resolved one of the major issues the company was facing. 


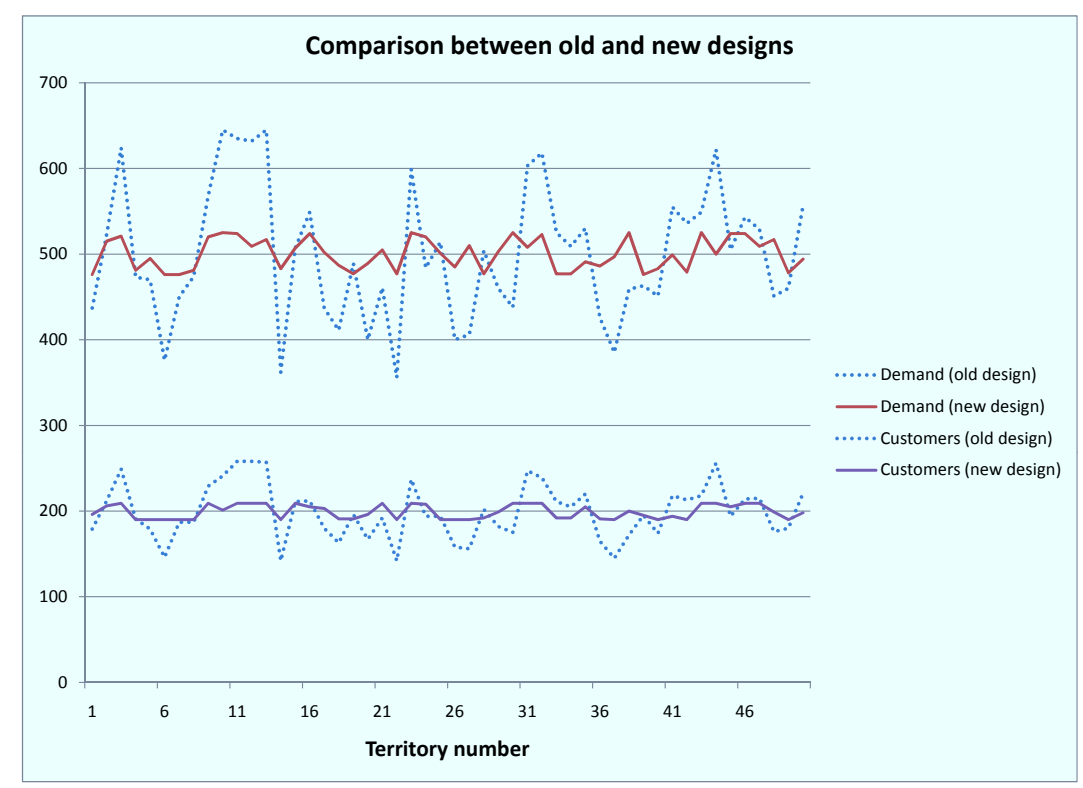

Figure 2: Comparison between territory size (number of customers and product demand) between old and new plan.

To illustrate this, we present a comparison of the original plan with the new plan obtained by the developed tool in a 5000-BU, 50-territory instance. Figure 2 shows the size distribution with respect to the two activity measures (number of customers and product demand) under these two scenarios. As can be seen, the disparity in size among the territories under the previous design was very large with respect to both activities. This significantly contrasts with the new plan.

Figure 3 displays the same comparison, but this time plotting the relative deviation of each territory with respect to the ideal target in numbre of customers (top) and product demand (bottom). Under the new plan, as can be seen this deviation falls within $5 \%$.

Figure 4 and 5 display the graphical solution of the previous design and the new design (under a tolrenace equal to 0.05), respectively. This is a feasible solution satisfying all of the planning constraints. The legend besides the graph indicates the number of BUs contained in each territory. The instance was solved in a few minutes with the developed tool. 

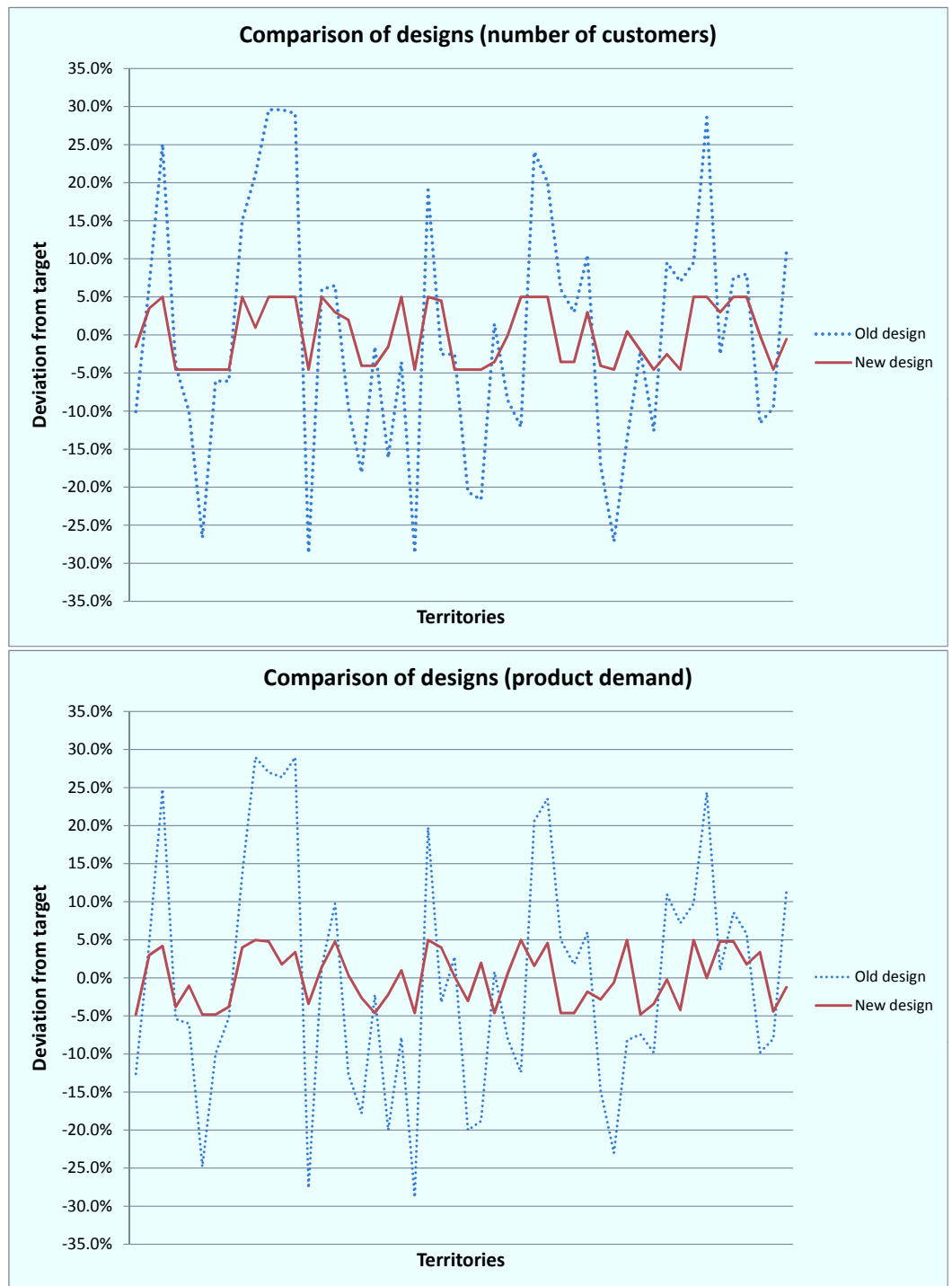

Figure 3: Comparison between old and new design in terms of deviation from ideal target with respect to number of customers (above) and product demand (below). 


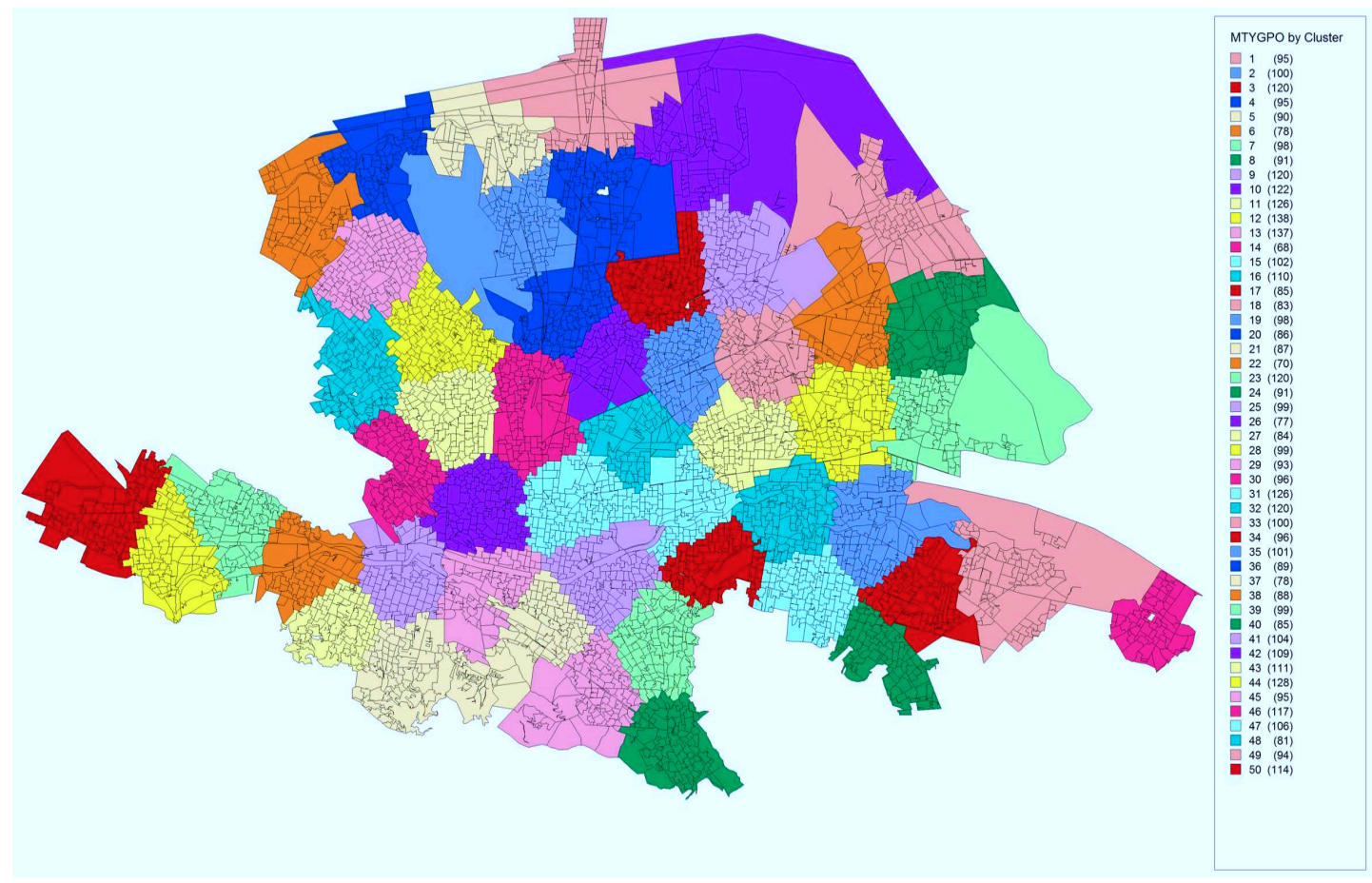

Figure 4: Previous territory design in Monterrey.

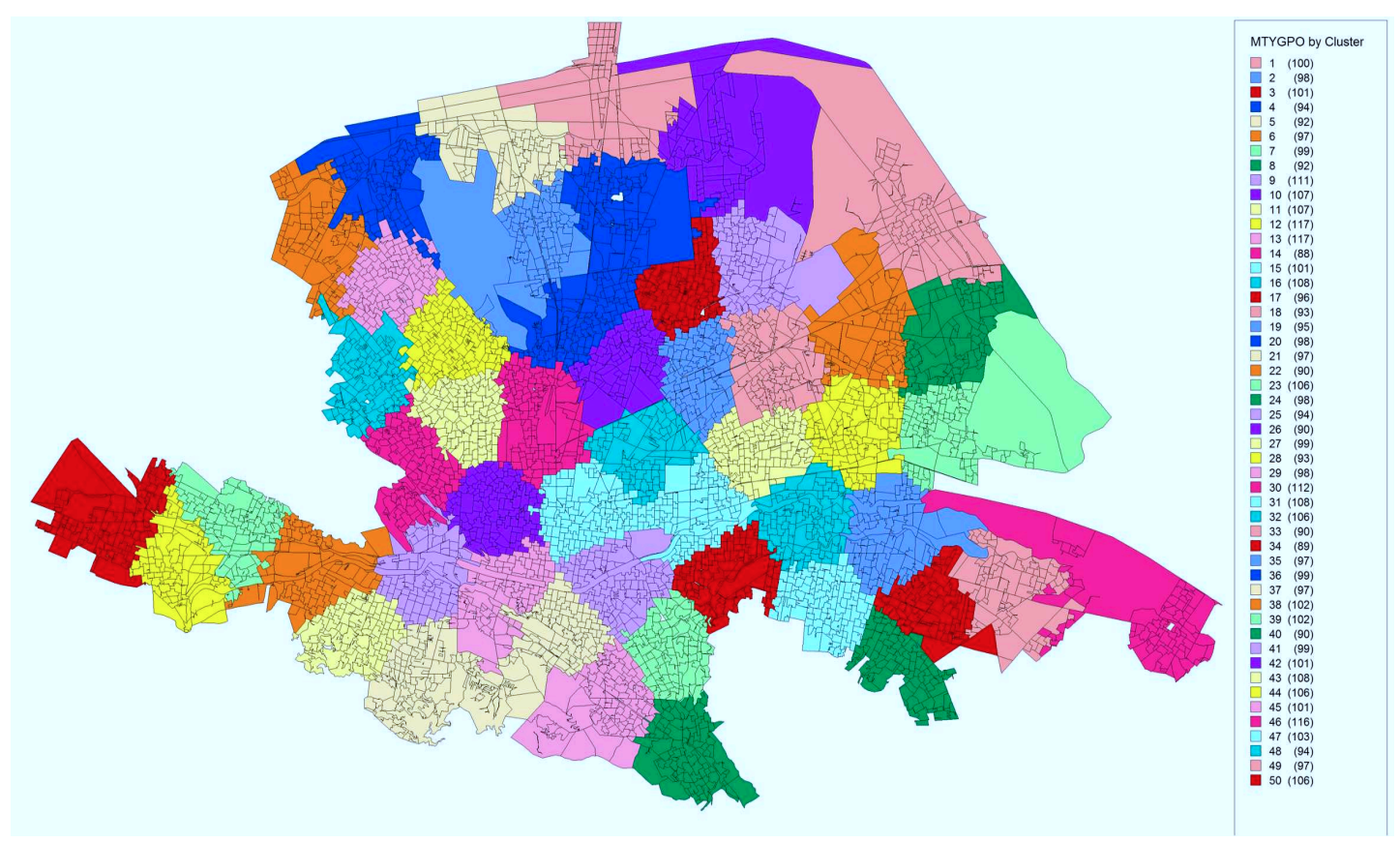

Figure 5: New territory design in Monterrey (tolerance $=5 \%$. 
Our tool is also usefull to assess the natural trade-off between balancing and territory compactness. To illustrate this, we solved the 5000-BU 50-territory problem instance for different values of the user-defined tolerance $\left(\tau^{a}=0.05, \ldots, 0.10\right.$ for $\left.a=1,2\right)$. Figure 6 shows the results of this experiment. It can be observed how these measures are in conflict, that is, as one a tighter (smaller) value for the balance constraint allowed tolerance is attempted, the dispersion measure value increases, and viceversa. Therefore, the developed method becomes a very valuable tool for evaluating different solutions in terms of these two factors.

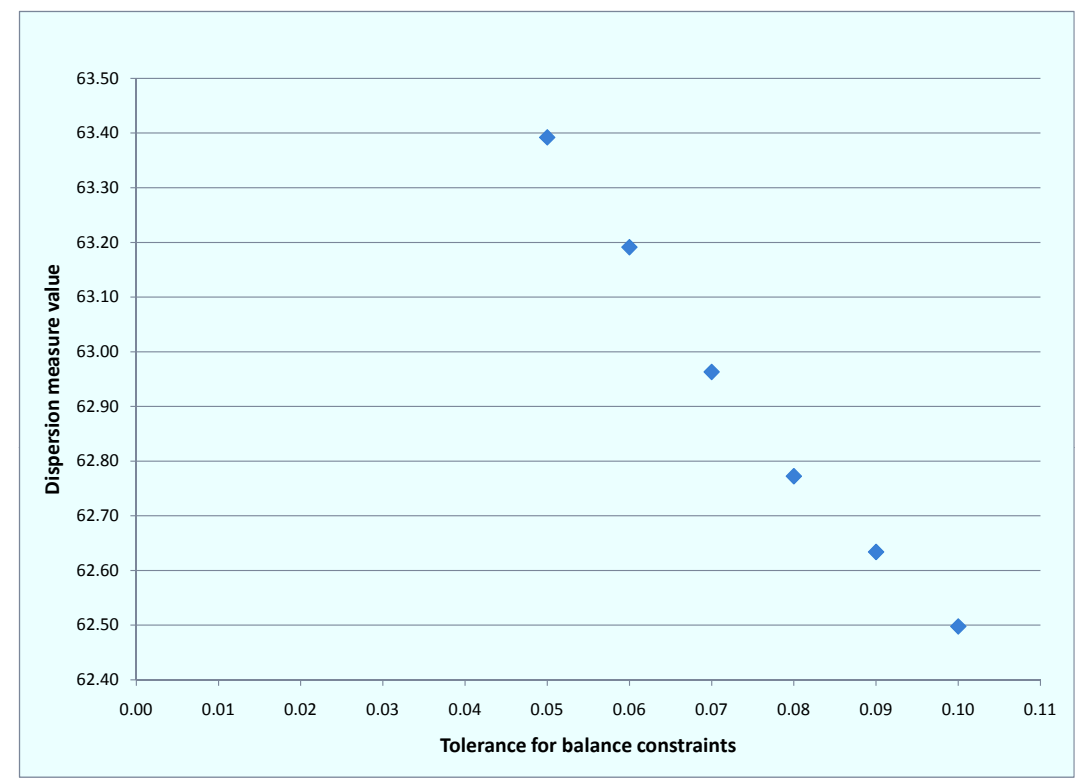

Figure 6: Trade-off between balance constraint tolerance and dispersion measure.

\section{Benefits}

We integrate our model into an advanced interactive tool based on the MAPINFO (C)application. Thus, we achieve a practical functionality to the end-users. This GIS environment can be used in different contexts. At the operational level, it represents a valuable tool to quickly produce and deploy different solutions. At the tactical level it can be used to simulate alternative scenarios and evaluate the impact of changes in territories. It is important to point out the interest of the end users about how our model can easily take the already existing territories into account. Particularly, the model is prepared to consider any prescribed and forbidden assignment of BUs. This means that one can impose some fixed territory centers or BUs allocations to territory centers, which have to be taken into account. Thus all these features can be extended for any case when some territory information is present at the be- 
ginning of the planning process. The issue of territory realignment is an important feature for the company because it is crucial for customer satisfaction. Thus, the company evaluates how our model efficiently accommodates for system changes like customer's additions or dropouts trying not to disrupt the previous design considerably.

From the business standpoint, our TDP application was developed and implemented at Embotelladoras ARCA in order to optimize the distribution operation to the end customers. During the past few years, the firm was interested in developing a better territory and routing plan for the distribution operation to end customers. In fact, this is the first operation research (OR) application that has been implemented in ARCA. The company point out that the overall results have been very positive. The company top management recognize that features included in the OR model implemented are truly outstanding. The project was a major challenge, requiring a great deal of thought and effort. The first plans for territory design suggested by the optimization model were implemented in mid 2010. Throughout the ramp-up and launch of the project, those plans for distribution operation were analyzed. Sometime after, the project has resulted in a significant increase in productivity and direct savings to the firm. We can list some of the benefits that the company has achieved within this project.

- Identification now of a rational set of activity measures to target and balance on each truck resource. This results on an optimal fleet of trucks, drivers and sales people.

- An increase in efficiency and effectiveness on the planning process required to set up territory and route designs. The typical fully-manual planning process time was reduced from 2 weeks to less than an hour using the new OR application. This permitted the company to refine its capacity each season on a dynamic basis. As a result, the company achieves an optimal capacity to attend demand on each territory with an optimization of 30 delivery routes on the Monterrey metropolitan area. This represents a $15 \%$ reduction from the original number of routes.

- Streamline truck capacity to align it to a new end customer distribution strategy. The added throughput allows the firm to defer investments on trucks and other equipments that were originally allocated. The save on investments for trucks was about $8 \%$ of the entire fleet.

- Identification and implementation an optimal cost of service depending on each route model type. This allowed the firm to set an optimal frequency for customer delivery operations. This means less travel time between customers and $5 \%$ increase in volume delivered per route per day. 
- No more territory overlapping. As a result of the connectivity constrained featured model, there are no more territory overlaps and the territories are now mores appropriately defined in geographic terms. It is now easy to decide which sales worker would be responsible when new customers appears (and for dropouts too). They have been able to better define areas of responsibility and loading.

- Better territory compactness. As a result of our compactness objective featured model, the territories are more compact so the total travel time decreased, improving the productivity of the distribution people. According to the compactness measurement, the managers decided to rationalize the number of trucks available to the distribution people.

- Improved balancing with respect to the three activity measires. Our model deals with a small territory tolerance on lower and upper bounds for the three activity measures around $5 \%$. The "after alignment" structure is much better balanced than the former one. The standard deviation of the "number of customers per territory" or the "level of workload for each salesman" decreased $24 \%$ in average. This alignment allows making an increase in the level of service to the end customers on the marketplace. An increase on sales at the $3 \%$ is estimated as direct benefit of the new territory alignment.

Besides all these business benefits, the new OR model will allow the company to speed up some others "Route to Market" initiatives which are of special interest among Coca Cola bottlers around the world. The proposed model approach can extend the basic model to address different specific business rules or additional planning criteria. Some of these can be easily modeled as activity measures on the BUs. Overall, we have provided a very valuable tool for a more efficient territory design planning according to the company business requirements. Our model is prepared to deal with very large instances, even larger than 10000 BUs. Nowadays, our model is being used by the firm to obtain a business solution with significant benefits.

\section{Final Remarks}

In this paper, we have addressed a territory design problem as a critical component of the operational planning process in sales and services companies. Many logistics problems found in service industry can be modeled as a TDP. TDPs are multidisciplinary and have been widely studied in the operations research literature. However, solving a real world TDP possesses a significant challenge for both researchers and practitioners. A real world 
TDP includes many business rules and logic that are beyond those addressed in mathematical models in literature. In particular, there are some business rules such as territory connectivity that is fairly complex to deal with. A particular emphasis is given to a business application case at Embotelladoras ARCA. With a real world application from the service industry, we present a rich featured TDP model. We include some extensions that are very common to some of the problems encountered in industry. Because of the characteristics of a TDP, it is also challenging to obtain solutions within a reasonable computational time based upon the concrete business requirement. Furthermore, field people who are going to deploy the solution of a TDP may have to pay more attention to the feasibility of the solution in practice than a pure optimal solution in terms of mathematics.

Our TDP instance is motivated by a real world application in the soft drink industry. In particular, it is of interest to deal with very large scale instances. Several different objectives and constraints in the territory design process are identified and discussed. In order to tackle these simultaneous and conflicting objectives, a MILP-based solution framework has been developed to accommodate the particular business requirements. The proposed framework incorporates some algorithmic strategies that allowed to solve the problem more efficiently. Our implementation is based on a cut generation strategy that solves a relaxed model (relaxing the exponential number of connectivity constraints) and then iteratively identifies and adds violated cuts by solving an easy separation problem.

The proposed model not only addresses the difficulties embedded in the typical TDP problem but also some practical concerns about pre-defined and/or forbidden joint assignments of BUs. Pre-assigned or forbidden requirements arise from business issues such as territory realignment. From the practical standpoint, the issue of territory realignment focuses on how the model could efficiently accommodate for changes like customer additions or dropouts trying not to disrupt the previous design considerably. With respect to our industrial experience as well as the end-users thoughts at Embotelladoras ARCA, we believe that our model can be applied in quite different settings such as sales territories, locations of new stores in a chain, and delivery areas for distribution. In summary, our model and approach are capable of solving very large-scale real world TDP instances, and has been successfully used by the company resulting in many benefits.

Acknowledgements: The second author kindly acknowledges the financial support of the Mexican National Council for Science and Technology (grant SEP-CONACYT 48499Y), and Universidad Autonoma de Nuevo Leon through its Scientific and Technological Research Support Porgram (grant UANL-PAICYT CE012-09). 


\section{References}

[1] R. F. Deckro. Multiple objective districting: A general heuristic approach using multiple criteria. Operational Research Quarterly, 28:953-961, 1977.

[2] J. C. Duque, R. Ramos, and J. Suriñach. Supervised regionalization methods: A survey. International Regional Science Review, 30(3):195-220, 2007.

[3] J. Kalcsics, S. Nickel, and M. Schröder. Toward a unified territorial design approach: Applications, algorithms, and GIS integration. Top, 13(1):1-56, 2005.

[4] R. Z. Ríos-Mercado and E. A. Fernández. A reactive GRASP for a commercial territory design problem with multiple balancing requirements. Computers $\&$ Operations Research, 36(3):755-776, 2009.

[5] M. A. Salazar-Aguilar, R. Z. Ríos-Mercado, and M. Cabrera-Ríos. New models for commercial territory design. Networks and Spatial Economics, 2011. (Forthcoming, doi: 10.1007/s11067-010-9151-6).

[6] M. A. Salazar-Aguilar, R. Z. Ríos-Mercado, and J. L. González-Velarde. A biobjective programming model for designing compact and balanced territories in commercial districting. Transportation Research Part C, 2010. (Forthcoming, doi: 10.1016/j.trc.2010.09.011).

[7] M. A. Salazar-Aguilar, R. Z. Ríos-Mercado, and J. L. González-Velarde. GRASP strategies for a bi-objective commercial territory design problem. Journal of Heuristics, 2011. (Forthcoming, doi: 10.1007/s10732-011-9160-8).

[8] L. Vargas-Suárez, R. Z. Ríos-Mercado, and F. López. Usando GRASP para resolver un problema de definición de territorios de atención comercial. In M. G. Arenas, F. Herrera, M. Lozano, J. J. Merelo, G. Romero, and A. M. Sánchez, editors, Proceedings of the IV Spanish Conference on Metaheuristics, Evolutionary and Bioinspired Algorithms (MAEB), pages 609-617, Granada, Spain, September 2005. In Spanish.

[9] A. A. Zoltners. A unified approach to sales territory alignment. In R. P. Bagozzi, editor, Sales Management: New Developments from Behavioral and Decision Model Research, pages 360-376. Marketing Science Institute, Cambridge, 1979.

[10] A. A. Zoltners and P. Sinha. Sales territory alignment: A review and model. Management Science, 29(11):1237-1256, 1983.

[11] A. A. Zoltners and P. Sinha. Sales territory design: Thirty years of modeling and implementation. Marketing Science, 24(3):313-331, 2005. 


\section{Appendix. TDP Model}

Let

- $V$ be the set of BUs, $|V|=n$;

- $E$ be set of edges representing adjacency between BUs;

- $V_{c}$ be the set of territory centers, $\left|V_{c}\right|=p$;

- $A=\{1,2,3\}$ be the set of node attributes corresponding to number of customers $(a=1)$, product demand $(a=2)$, and workload $(a=3)$;

- $c(k)$ denote the index of center of territory $k$;

- $d_{i j}$ be Euclidean ditance beween BUs $i$ and $j$,

- $w_{i}^{a}$ be the value of attribute $a \in A$ in $\mathrm{BU} i \in V$;

- $w^{a}\left(V_{k}\right)=\sum_{i \in V_{k}} w_{i}^{a}$, the size of territory $V_{k} \subset V$ with respect to activity ain $A$;

- $\mu^{a}=w^{a}(V) / p$ the average target size of activity $a \in A$;

- $N^{i}=\{j \in V:(i, j) \in E \vee(j, i) \in E\}$ be the set of nodes which are adjacent to node $i ; i \in V$;

- $H_{d}$ be the set that contains all pairs of BUs that must be assigned to different territories;

- $F^{i}$ be the pre-specified subset of BUs associated to center $i$ from an existing plan;

- $q_{i j}$ be the penalty term for assigning unit $j$ to center $i \in V_{c}$, equal to $0.5 d_{i j}$ if $j \in F^{i}$, and equal to 0 , otherwise;

- $\tau^{a}$ be the user-specfied tolerance parameter for activity $a \in A$;

The decision variables are deifined as $x_{i j}=1$ if the BU $j$ is assigned to territory with center in $i$, and 0 otherwise; $i \in V_{c}, j \in V$. Note that $x_{i i}=1$ implies that unit $i$ is a territory center.

\section{Allocation Model (AM)}

$(\mathrm{AM}) \min \sum_{\substack{i \in V_{c} \\ j \in V}} d_{i j} x_{i j}+\sum_{\substack{i \in V_{c} \\ j \in F^{i}}} q_{i j}\left(1-x_{i j}\right)=f(x)$

subject to

$$
\sum_{i \in V_{c}} x_{i j}=1 \quad j \in V
$$




$$
\begin{array}{rlrl}
\sum_{j \in V} w_{j}^{a} x_{i j} \leq\left(1+\tau^{a}\right) \mu^{a} & & i \in V_{c}, a \in A \\
\sum_{j \in V} w_{j}^{a} x_{i j} \geq\left(1-\tau^{a}\right) \mu^{a} & & i \in V_{c}, a \in A \\
\sum_{j \in \cup_{v} \in S^{v} \backslash S} x_{i j}-\sum_{j \in S} x_{i j} \geq 1-|S| & & i \in V_{c} \\
& & S \subset V \backslash\left(N^{i} \cup\{i\}\right) \\
x_{i j}+x_{i h} & \leq 1 & & i \in V_{c},(j, h) \in H_{d} \\
\sum_{i \in V} \sum_{j \in F^{i}} x_{i j} \geq \alpha\left|\cup_{i} F^{i}\right| & & \\
x_{i j} & \in\{0,1\} & & i \in V_{c}, j \in V
\end{array}
$$

Objective (1) incorporates a term that measures territory dispersion and a term that favors the assignment of a subset of units from existing plan. Constraints (2) guarantee that each node $j$ is assigned to a territory. Constraints (3)-(4) represent the territory balance with respect to each activity measure as it establishes that the size of each territory must lie within a range (measured by tolerance parameter $\tau^{a}$ ) around its average size. Constraints (5) guarantee the connectivity of the territories. Note that there is an exponential number of such constraints. The disjoint assignment is represented by constraints (6). Constraints (7) assure that at least a minimum number of BUs from existing plan is assigned, where $\alpha$ is a user-specified parameter usually set to 0.10 to 0.20 in practice.

Allocation Model Relaxation (AMR): Given the exponential number of connectivity constraints (5) for our solution procedure we consider the relaxation of these constraints and called this relaxed model AMR. Note that the integrality constraints are kept. 